\title{
Le temps bref des contes à rire
}

\author{
Josiane Bru \\ LISST/Centre d'anthropologie sociale \\ Josiane.Bru@ehess.fr
}

\begin{abstract}
RÉSUMÉ
Dans la partie introductive du catalogue français des contes d'animaux, Marie-Louise Tenèze pose la question: "Qu'est-ce qu'un conte? ». Afin de resserrer son propos sur ce genre narratif dans son ensemble, elle établit une série de critères (internes et externes) qui permettent d'en écarter certains récits ou dires de tradition orale relevant d'autres catégories, non répertoriées en tant que telles dans la classification internationale des contes populaires : légende, récit étiologique, mimologisme-récit, anecdote. Ce sont pour la plupart des récits brefs.

Partant d'exemples issus de la section Anecdotes and Jokes du catalogue international, on posera la question de la temporalité de ces récits brefs pour envisager le conte dans le cadre plus global de la "contée », selon le terme employé par les conteurs contemporains pour désigner le temps et les modalités de la performance.
\end{abstract}

\section{MOTS CLÉS}

conte populaire, contes facétieux, récit, temps du récit, organisation narrative

\section{RESUM}

A la introducció del catàleg francès de les rondalles d'animals, Marie-Louise Tenèze planteja la qüestió: «Què és una rondalla?». Per tal de reforçar les seves observacions sobre aquest gènere narratiu en el seu conjunt, estableix una sèrie de criteris (interns $i$ externs) que permeten d'excloure certs relats o narracions de tradició oral d'altres categories que no figuren com a tals en la classificació internacional de les rondalles. Es tracta sobretot de narracions curtes: llegenda, relat etiològic, mimologisme-relat, anècdota.

Intentarem examinar el vincle que els contes-tipus aplegats a la secció Anecdotes and Jokes de la classificació internacional mantenen amb la brevetat, tant des del punt de vista del contingut com del de la forma i del temps d'execució.

PARAUlES CLAU

rondalla, facècia, relat, temps del relat, organització narrativa 
Josiane Bru

\begin{abstract}
In the introduction of the French catalogue of animal tales, Marie-Louise Tenèze asks the question: "What is a tale?". To support her observations on this narrative genre as a whole, she establishes a series of internal and external criteria that allow her to exclude certain stories in the oral tradition from other categories that do not figure as categories in the international classification of folktales. Mostly these are short stories, legends, etiological stories, mimological stories and anecdotes.

We will examine the link between the story-types gathered in the Anecdotes and Jokes section of the international classification and their brevity in terms of content form and time of execution.
\end{abstract}

\title{
KEYWORDS
}

Folktale, Joke, report, time of report, narrative organization 
DANS LA PARTIE INTRODUCTIVE du troisième volume du catalogue des contes populaires français, Marie-Louise Tenèze pose la question : "Qu'est-ce qu'un conte? » Étendant l'interrogation qu'elle porte sur la catégorie des contes d'animaux (Tenèze I976:3-4) à l'ensemble de ce genre narratif jamais défini dans son ensemble, elle établit une série de critères externes et internes qui tendent à en écarter certains. Il s'avère que les catégories dont relèvent ces récits au statut problématique, mis à l'écart du genre désigné par le terme « conte » pour des raisons diverses, rassemblent une majorité de récits brefs. Les principaux sont:

- la légende et le récit étiologique, du fait de leur imbrication avec le système des croyances qui les exclut du domaine de la fiction.

- l'anecdote, en raison de son organisation narrative particulière: deux parties inégales dont la seconde, écrit-elle, très brève, ferme l'anecdote sur ellemême, coupant court à tout prolongement ou relance:

L'anecdote est par définition un récit autonome; il est impossible de la reprendre, de la continuer, même de lui ajouter quoi que ce soit; elle est semblable à la pièce d'un feu d'artifice: une fois que celle-ci, bien amorcée, a éclaté, c'est fini. La moindre « chaîne » du loup et du renard nous convaincra immédiatement qu'une autonomie si radicale oppose inéluctablement anecdote animale et conte d'animaux (Tenèze i976: 43).

Portant sur la forme, la remarque vaut pour d'autres catégories de récits où l'on peut même retrouver des actions identiques portées par d'autres personnages (l'homme et le diable du conte-type ıozo se partagent la récolte de la même manière que les deux animaux du T. 9).

En m'appuyant sur l'étude qu'en fait M.-L. Tenèze, je poserai donc la question de la composition de ces récits oraux à partir de la seule catégorie de la classification internationale dont les protagonistes sont des humains. ${ }^{I}$ Dénommée Schwänke dans le catalogue d'Aarne en I9Io, elle devient Jokes and Anecdotes dans l'Aarne-Thompson puis Anecdotes and Jokes dans l'ATU qui, en inversant les termes, prend acte de la grande vitalité de l'anecdote cependant que de leur côté les collecteurs de la seconde moitié du $\mathrm{xx}^{\mathrm{e}}$ siècle pointent le foisonnement des récits facétieux dans les répertoires de leurs informateurs. Paul Delarue et MarieLouise Tenèze, auteurs du catalogue des contes populaires français, utilisent l'expression Contes facétieux et anecdotes.

I. Les rares personnages surnaturels présents dans des récits classés entre les T. I200 et T. 1999 n'ont pas de rôle significatif. 


\section{Histoires brèves... en série}

La majorité des contes-types de la section Contes facétieux et anecdotes sont des récits brefs, limités à une seule action mettant en présence un nombre très restreint de personnages, généralement engagés dans des oppositions binaires: mère et fils dans les contes de Jean-le-Sot, mari et femme dans les histoires de couples, notable ou clerc et paysan dans d'autres. On a parfois un protagoniste collectif, un groupe de villageois par exemple dans de nombreuses béotiana comme dans les contes-types T. I245 ou T. I250 par exemple.

Sunlight Carried in a Bag (Basket, Sieve) into the Windowless House (T. I245). Dans des versions françaises, des villageois franchissent toutes les nuits la montagne pour aller chercher le soleil (le jour) et le ramener le lendemain matin.

The Human Chain (T. I250). Un groupe d'idiots décident de faire une chaîne pour sonder la profondeur d'un puits en s'accrochant l'un à l'autre. Le premier, qui s'accroche à une poutre posée en travers du puits, se lâche pour cracher dans ses mains et tous tombent au fonds.

Nombre de ces histoires brèves, parce qu'elles se présentent de façon isolée dans les répertoires, sont indexées de manière autonome dans le système de Aarne et Thompson qui leur assigne un numéro de conte-type. ${ }^{2}$ Ces histoires, anecdotiques par leur légèreté aussi bien que par leur brièveté, se retrouvent intégrées dans des narrations plus longues dont elles constituent un épisode. Ainsi les villageois naïfs du T. I245 peuvent-il être les clients du jeune homme qui hérite d'un coq dans des versions du T. I650 : il le leur vend en affirmant que l'animal fait venir le jour et qu'ils n'auront ainsi plus besoin d'aller le chercher avant l'aube.

Abstraction faite de la technique très rôdée consistant à étoffer le récit par des détails ou commentaires qui ne changent rien à son caractère mono-épisodique, ${ }^{3}$ une partie des contes facétieux "longs " sont ainsi des assemblages de petits contes courts. Michèle Simonsen (2003: 258-259) en a relevé quelques-uns, parmi les plus connus.

De quoi sont faits ces assemblages? Qu'en est-il de cette longueur qui, nous dit M.-L. Tenèze, « est atteinte de tout autre façon que dans le conte merveilleux »? (Tenèze I976 : 26). Les configurations sont diverses et nous en examinerons quelques-unes à partir de versions qu'elle et Paul Delarue, puis moi-même, avons répertoriées en vue du catalogue français. ${ }^{4}$

2. Comme pour les contes d'animaux « une partie des numéros, des 'types' [...] ne se rencontrent que sous forme de parties d'oeuvres plus longues, sous forme, si l'on convient d'appeler tel la partie insécable d'un ensemble constitué par un couple de fonctions, d'épisode » (Tenèze i976: 58).

3. Étoffer un récit est une technique de l'art narratif indifféremment utilisé pour faire durer le plaisir du conte, quel qu'il soit, y comprit les contes merveilleux dans les intervalles desquels les conteurs parfois glissent de petites parenthèses ludiques (contes d'animaux ou autres récits brefs). Alors que, n'ayant jamais entendu de conteur issu de la tradition populaire, j'avais beaucoup de difficultés à en saisir la grande liberté, je m'étais étonnée de telles «parenthèses » dans la version du Roi des corbeaux (T. 425) donnée d'après J. F. Bladé par la conteuse occitane Thérèse Canet, au Théâtre de la Digue, en I998.

4. En attendant de fixer la totalité des intitulés des contes-types pour la partie du catalogue français concernant les T. Iooo et suivants, je donne seulement les titres anglais de l'ATU 


\subsection{Des chaînes et cycles de récits brefs}

Une première sorte de contes facétieux « longs » est formée par juxtaposition d'actions indépendantes, en ordre et de présence plus ou moins aléatoires. Les histoires de Jean-le-Sot en donnent un exemple caractéristique comme le montrent les T. I68I et T. I696. A la lecture des recueils, il est difficile de savoir si elles étaient contées à la suite ou séparément. Quand elles ne sont pas enchaînées par le conteur en un seul et même récit, scandé par des " une autre fois... », elles forment du moins un cycle homogène autour d'un même personnage qui sert de prétexte à la série dans laquelle d'autres courtes histoires semblables peuvent être intégrées comme autant d'épisodes. Ce qui tient ensemble les divers épisodes, c'est le plus souvent l'identité du « héros », stigmatisé par son nom. La cohérence de l'ensemble n'a pas d'importance puisqu'on voit parfois le conteur reprendre son récit après qu'une mésaventure ait entraîné la mort de son personnage. Parfois aussi il en inverse la caractéristique principale : guéri subitement de sa sottise, l'idiot devient « fin ». Cette mutation soudaine et souvent immotivée ouvre à une autre série d'aventures sans pour autant interrompre le cours du récit (Bru, 2007-2008). Le héros meurt aussi parfois, ce qui est une autre façon de conclure, comme meurt la femme obstinée noyée par son mari qu'elle traite jusqu'à la fin de pouilleux (T. I365C: The Wife Insults the Husband as Lousy-head).

Comme l'indique Jean-Noël Pelen (I994: 23I) : « Les cycles constitués, autour d'un personnage ou de plusieurs, relèvent la plupart du temps ou en grande partie d'une thématique précise et d'une seule, incarnée par le personnage ». Celui-ci peut être une figure locale ou historique qui, en raison d'un trait de son caractère réel (esprit ou sottise, avarice, vantardise...) devient le héros de petits contes populaires attribués dans d'autres régions à des protagonistes différents ${ }^{5}$. Certains curés hauts en couleur ou des personnages prestigieux sont ainsi les acteurs plus ou moins involontaires de ces séries d'histoires plaisantes. ${ }^{6}$

\subsection{Des associations stables de contes-types}

Suivant les pays ou régions, certaines de ces histoires brèves généralement séparées par ailleurs, sont associées de façon stable pour former un conte plus long. ${ }^{7}$ C'est le cas en France des contes-types T. I450 (Clever Elsie) et T. I384 (The Hus-

(Cf. Bru 2005). La documentation constituée par P. Delarue et M. L. Tenèze, conservée à Paris, au Musée National des Arts et Traditions Populaires, est transférée au MNATP/MUCEM à Marseille. Une copie des fichiers visant à compléter le Catalogue est déposée au LISST/ Centre d'Anthropologie, à Toulouse où sont rassemblées les nouvelles références.

5. Il en est ainsi du Voleur d'Alpuech en Aubrac (Tenèze I975: I2O-I2I et I978: I4I-I46) mais aussi d'Henri IV, de Napoléon et bien d'autres.

6. Pour rester dans ma région d'origine je citerai seulement la série de «bessonadas » (prononcer : béssounados), histoires attribuées à l'abbé Bessou, auteur plusieurs recueils de contes très prisés en Rouergue (Aveyron), connu pour ses réparties cinglantes. Le poète toulousain Goudouli (Peire Godolin), auquel on doit une renaissance de la langue d'oc au début du XVII siècle, était réputé pour ses bonnes farces que l'on trouve rapportées jusque dans les journaux populaires du début du XIXe siècle sous le nom de «godolinadas » (pr. goudoulinados).

7. C'est l'équivalent, sur le mode de la juxtaposition, de la combinaison stable de contes merveilleux qui forment sur une aire culturelle, un écotype. Carme Oriol me signale la combinaison T. $3 \mathrm{IIB}^{*}(A T U)+\mathrm{T}$. I655, très stable dans les répertoires catalans. 
band Hunts Three Persons as Stupid as his Wife, incluant dans la définition de l'ATU l'ancien T. I37I*). Il en est de même pour les T. I240 ATU (Cutting Off the Branch) et T. I3I3 AT (The Man who Thought HimselfDead) que Delarue proposait de placer ensemble sous le seul T. I240 dans le catalogue français en raison de la fréquence de leur enchaînement. Une association d'idées, basée sur un mot, une simple assonance ou autre rapprochement lié à la langue ou autre référence collective peut être à l'origine de cette configuration et de sa transmission.

\subsection{Le modèle de la Randonnée}

Les Randonnées (T. 2000-T. 2100: Cumulative Tales) forment une catégorie particulière. ${ }^{8}$ Plus que des contes à proprement parler, ce sont de quasi-récitations admettant une certaine variabilité, comme le sont beaucoup de Formula Tales. Il s'agit de la répétition compulsive de la même action avec, à chaque fois, le léger décalage introduisant un nouvel élément. Dans les quelques contes facétieux construits sur ce modèle, la forme fixe de la randonnée disparaît en partie au profit de l'action racontée et du sens que prend la remise en ordre finale.

Dans le conte de l'échange profitable, ou conte de la lentille (T. I655: The Profitable Exchange) un homme trouve une lentille et demande qu'on la lui garde afin de ne pas la perdre durant la nuit. Une poule la mange et il exige d'emporter la poule, puis le jour suivant il emmène le cochon qui a mangé la poule, l'âne qui a tué le cochon etc. Il augmente ainsi par chantage la valeur de son avoir. Il obtient en dernier lieu d'emmener une jeune fille qu'on lui demande de cacher dans un sac afin que personne ne s'en aperçoive. Lorsque, arrivé dans un lieu isolé, il croit pouvoir l'embrasser, il est mordu par une chienne boiteuse qu'on a mise à sa place et qui lui emporte le nez.

Un même thème peut donc être traité en randonnée ou en conte suivant que le narrateur reprend ou non à l'identique la phrase antérieure, allongée à chaque étape.

\subsection{Le modèle du conte merveilleux}

Quelques contes facétieux longs sont construits sur le modèle des contes merveilleux ou, du moins, en empruntent les principaux éléments caractéristiques : le motif du départ, la «triplication » des personnages et des épreuves, la réussite spectaculaire du plus jeune...:

Dans le conte du Fin voleur (T. I525: The Master Thief), trois frères pauvres quittent la maison pour apprendre un métier. Lorsqu'ils se retrouvent au bout d'un an, les deux aînés ont pris des voies respectables, mais le plus jeune est devenu maître (fin) voleur. Sommé de prouver sa finesse dans cet art en commettant une série de larcins apparemment irréalisables, il vient à bout de la série d'épreuves qui lui sont imposées par le maître ou seigneur du village.

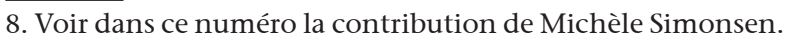


L'essentiel du conte merveilleux pourtant n'y est pas puisque le héros reste identique à lui-même, prêt à d'autres aventures où il exercerait sans fin son art. Un glissement est cependant possible, quoique rare, vers une clôture positive et définitive du récit. C'est le cas dans la version que donne Nannette Lévesque à Victor Smith, au milieu du XıX ${ }^{\mathrm{e}}$ siècle (Tenèze et Delarue éds. 2000: 254 et 260-264). Le héros de cette variante auvergnate particulière transforme en dot les gains que lui procure sa réussite dans la dernière épreuve (subtiliser le trésor du maître) et s'établit honnêtement. Il achète une ferme et se marie, à la manière des héros de contes merveilleux. La proximité formelle du conte merveilleux ne suffit pas à expliquer une telle transformation du conte, c'est ici la vision du monde de la conteuse, sa foi chrétienne profonde, qui induit le changement d'orientation du récit et son aboutissement.

Parfois l'emprunt formel au conte merveilleux est plus limité, plus superficiel:

Dans le T. I650: (The Three Lucky Brothers) chacun des trois fils d'un homme pauvre fait fortune à son tour en vendant à des naïfs sa minuscule part d'héritage (le chat, la faucille et le coq). C'est le plus jeune qui gagne le plus d'argent dans la vente.

Le motif initial des trois frères relie trois épisodes indépendants, trois unités narratives très simples, constituées chacune d'une seule action, que nous avons évoquées plus haut. C'est ici le simple hasard des rencontres qui permet à chacun de s'enrichir sans pour autant lier structurellement les trois épisodes qui peuvent être contés séparément, comme autant de récits brefs. La petite série pourrait tout aussi bien entrer dans une configuration l'associant aux T. I45O et T. I384 cités ci-avant (un seul garçon possédant les trois « objets ", effrayé par la sottise de ses « clients », revenant ensuite épouser sa fiancée idiote.

\subsection{L'influence de l'écrit et la proximité du conte-nouvelle}

Certains contes facétieux multi-épisodiques peuvent paraître plus complexes parce qu'ils abordent des thèmes développés plus sérieusement dans les contesnouvelles, comme l'injustice et la spoliation, où les conflits sont heureusement résolus. Mais alors que dans ces derniers la protagoniste (c'est généralement une fille ou jeune femme), prend le départ et affronte une succession d'épreuves au terme desquelles elle se fera reconnaître et récupèrera sa place injustement perdue, le héros des contes facétieux (généralement juvénile et masculin) entreprend une série d'actions vengeresses sans commune mesure - en nombre et en cruauté - avec ce qu'il a subi.

Ex. T. I538 ATU (The Revenge of the Cheated Man. Previously: The Youth Cheated in Selling Oxen). Ayant fait le pari de soustraire à un garçon l'animal qu'il va vendre à la foire, deux filous (le plus souvent des moines) prétendent que la bête est d'une autre nature qu'elle n'est en réalité : que l'âne est une chèvre ou la vache un cochon par exemple. Tous trois conviennent de s'en remettre au jugement du premier homme qui passera. Un complice des deux filous se présente et donne son avis. Le garçon perd son animal et rentre chez lui, conscient d'avoir été berné. Il se venge par trois actions successives particulièrement cruelles jusqu'à ce qu'il récupère son bien et obtienne en surcroît une importante somme d'argent. 
Il en est de même dans le T. I539 (Cleverness and Gullibility), que l'ATU définit comme un «épisode humoristique composé de scènes populaires diverses et interchangeables $» .9$

Dans les versions françaises du T. I539 un homme ou un garçon se venge en vendant de faux objets magiques aux hommes qui l'ont trompé : le troisième objet est une flûte ou un sifflet qui ressuscite les morts. Ils tuent leurs épouses pour les impressionner et s'aperçoivent trop tard qu'ils ont été bernés par leur victime.

Contes nouvelles et contes facétieux ont pour caractère commun de traiter de personnages ordinaires, dont les agissements se situent très prosaïquement dans Ce Monde ${ }^{\text {Io }}$ mais ils y évoluent différemment. L'intention comique des uns, leur caractère facétieux, s'exprime par la multiplication des actions et leur démesure mais aussi, structurellement, dans l'ouverture du conte à d'autres épisodes dont chacun peut prêter à rire, introduisant ainsi une rupture dans le récit et l'écoute. Le conteur peut, si l'auditoire est réactif, en rajouter à sa guise sans jamais aboutir à une situation irréversible et socialement satisfaisante comme c'est le cas dans les contes merveilleux ou des contes-nouvelles.

La proximité de genre entre contes facétieux et contes-nouvelles est particulièrement évidente pour les récits oraux ayant des parallèles écrits : fabliaux et autres nouvelles. On peut légitimement penser que l'existence de ces versions fixes et pérennes aide au maintien de la forme longue et élaborée des variantes orales dont la vitalité est encore attestée au milieu du $\mathrm{XX}^{\mathrm{e}}$ siècle, qu'elle contribue à éviter leur fractionnement en autant d'histoires séparées. ${ }^{\text {II }}$ L'écrit fonctionnerait à la manière d'un schème présent dans la mémoire collective et soutenant l'oral de façon diffuse, comme un invisible tuteur. ${ }^{12}$

Ce peut être le cas, par exemple, pour les contes-types:

\section{T. I360C: Old Hildebrand,}

T. I535: The Rich and the poor Farmer (Unibos) dont le thème est attesté dès le $\mathrm{X}^{\mathrm{e}}$ siècle. ${ }^{\mathrm{I}}$

T. I730: The Entraped suitors, d'origine orientale selon l'E.M., présent dans les fabliaux français (Le lai de l'épervier).

9. «This episodic humorous tale is composed of various popular, interchangeable scenes (which often belong to Type I535, and therefore the types does not have well-defined limits ». (Uther 2004, II: 276). Cf. Bru (2005: 52-56).

Io. Je reprends ici la terminologie de Marie-Louise Tenèze (2004) pour qui l'opposition des êtres appartenant à Ce Monde (humain, terrestre) et ceux qui se situent dans l'Autre Monde (l'Au-delà, le Surnaturel) est le premier séparateur pris en compte dans son étude de l'organisation narrative des contes merveilleux français.

II. Les deux versions du Conte des Pères d'Aubrac, recueillies en 1964 et 1965 par M.-L. Tenèze (I975 : I45-I48 et I48-I5O) montrent l'actualité du T. I538 à cette époque, dans une zone rurale pauvre en grande partie dépendante d'un riche monastère.

I2. Cf. à un autre niveau la démonstration par Nicole Belmont (I985; I993) du rôle joué par le mythe latent d'Orphée dans la persistance de la forme très longue et complexe du contetype 3I3, La fille du diable.

I3. L'ATU indique: Traced to Versus de Unibove of the Ioth-IIth century, and popular since the I5th century (Sercambi, De bono fatto). Voir EM s.v. Unibos. 
T. I74I : The Priest's Guest and thee Eaten Chickens mis en vers par un anonyme français du XII ${ }^{\mathrm{e}}$ siècle (Les perdrix, ou Le dit des perdrix).

Le lien implicite avec des variantes écrites complexifie la réflexion en infléchissant le contenu du récit. En effet, on doit sans doute à cette proximité de l'écrit, destiné à un public autre que celui des conteurs populaires, une atténuation de l'action vengeresse qui reste alors relativement proportionnée à sa cause. Au contraire, dans le cas du T. I539 évoqué plus haut, la vengeance d'une simple duperie donne lieu au meurtre sans pitié des deux épouses innocentes. ${ }^{14}$

Les principes très pragmatiques de la classification internationale font que tout élément particulier, introduit ou mis en avant par le conteur, peut changer l'orientation d'un récit et le faire passer d'une catégorie narrative à une autre. Le conte se construit alors en arrière-plan et il faut attendre la fin pour en saisir la portée. C'est le cas du conte-type T. I4I5, Lucky Hans, lorsque la série des échanges désastreux menant le héros à la ruine se termine par sa victoire sur l'homme avec qui il a parié que sa femme ne se mettrait pas en colère. En raison de l'origine littéraire de ce conte, j'aurai recours à un autre exemple, plus probant, de changement d'accent, voire de catégorie, d'un conte dont le thème est classé comme facétieux.

Ex. T.I537: The Corpse Killed Five Times. Meurtrier par accident, un homme (ou son épouse) va déposer le cadavre devant la porte d'un voisin qui le prend pour un voleur (ou pour l'amant de sa femme) et le 'tue' avant de le porter devant la porte d'une autre personne et ainsi de suite, jusqu'au moment où le corps est déposé devant le presbytère: le curé croit que la femme est morte en venant se confesser et l'enterre comme il convient [autres solutions possibles].

Les versions françaises sont de simples chaînes: le meurtrier agit de sa propre initiative, les dépositaires successifs du cadavre n'ont aucun lien entre eux, aucun contact. La série de déplacements peut en principe être augmentée à loisir en intercalant une étape supplémentaire dans la série.

Un étudiant ivoirien en a confié à Lutz Röhrich (I990) une version recueillie en I980, dont il ne précise malheureusement pas s'il s'agit de la forme africaine de ce conte-type, d'une variation fréquente ou d'une originalité du conteur. L. Röhrich étudie par contre en détail l'inflexion que donne au conte ivoirien le recours à un personnage supplémentaire qui y prend une place centrale en mettant en perspective les éléments de la série: il s'agit d'une vieille femme indigente mais sage, que vont consulter tour à tour chacun des dépositaires successifs du cadavre. Celle-ci conseille à chacun de le placer devant la porte d'un autre habitant du village, qu'elle désigne chaque fois nommément. En échange ses conseils, elle obtient une gratification et comble ainsi progressivement son manque de moyens de subsistance.

Dans cette version, les images fortes du cadavre déplacé ancrent dans les esprits la loi de la communauté selon laquelle on ne doit pas laisser un vieillard dans le besoin. Rétablissant les liens de solidarité entre jeunes et vieux, le personnage

\footnotetext{
I4. Nicole Belmont (2004), dans une étude comparée des versions orales et des versions littéraires du conte-type 940 (Tenèze 2000: I67-I70 La Fille dédaigneuse et ses trois galants au cimetière) montre combien l'écrit respecte les limites de la bienséance et dans quelle mesure il influence le classement d'un thème qui est, dans ses versions orales, délibérément facétieux et 'licencieux'.
} 
de la vieille femme donne sens au conte et sa démarche limite la série de rebondissements qui cessent quand elle est assurée de pouvoir vivre confortablement. Le sort du cadavre est rapidement réglé après qu'il ait été finalement abattu par le gardien d'une plantation qui le prend pour un voleur. «Au lever du soleil le chef envoya des hommes chercher le corps qu'on enterra sans cérémonie » (Röhrich I990: 32I). Il en est de même dans les versions européennes où le cadavre est celui d'un homme (curé) amant d'une femme adultère, enterré par exemple par le boucher devant chez qui il est placé en dernier lieu. Dans les cas où le cadavre est féminin (ce peut être celui de la mère d'un niais, tuée par maladresse), le dernier destinataire du cadavre est un prêtre qui l'enterre religieusement. ${ }^{15}$

Les versions occidentales connues restent généralement ludiques et immorales. Elles se bornent à la répétition à l'identique d'une action furtive alors que le conte ivoirien au contraire se structure dans le temps grâce à l'introduction de l'actant médiateur qui lui confère une temporalité propre, chronologique et symbolique à la fois.

\section{Homologies temporelles}

Ainsi, en opposition à l'anecdote, genre narratif fondé sur la brièveté [...] le conte - qu'il soit merveilleux ou d'animaux, de l'un ou de l'autre des deux types d'organisation reconnus, est lui, consubstantiellement lié à la durée (Tenèze i976:59).

Le conte n'a pas pour seule fonction d'occuper le temps en le faisant passer: temps rêvé, temps de la trajectoire personnelle et/ou temps social, il s'y insère et le crée à la fois, de diverses façons qui, comme on l'a vu, forment des catégories en rapport avec la durée des récits. Une première homologie s’impose avec évidence entre la temporalité du conte (le temps dans lequel s'inscrit l'histoire racontée) et le temps qu'il faut pour le donner (la durée de la performance). Le passage progressif du héros ou de l'héroïne de l'enfance à l'âge adulte s'effectue lentement et le difficile franchissement des épreuves successives doit être exposé avec la même lenteur pour que le conte remplisse sa fonction initiatrice.

A ce temps long du conte merveilleux s'oppose, par sa structure comme par le caractère ponctuel de la plupart des actions relatées, le temps bref du conte facétieux. Cela est évident pour ceux qui exposent une action unique, isolée, mais, tout comme des pointillés ne suffisent pas à former une droite, c'est également vrai pour les contes composés de la façon que nous venons de décrire. L'apprentissage du voleur du T. I525 ne peut conduire qu'aux marges de la société et non à une intégration dans la communauté ; les trois frères du T. I650 vendent très prosaïquement les « objets » dont ils héritent et qui n'ont effectivement pas la fonction d'aides magiques susceptibles de faire avancer une quête initiatique. L'absence de projet constructif qui inscrirait le conte dans la durée est par ailleurs flagrante dans les T. I538 et I539 où le héros réagit de façon pulsionnelle et destructrice au préjudice subi. Ces contes ne dessinent pas un parcours, ils

I5. Dans un grand nombre de versions recensées pour le catalogue français, le mort est l'amant d'une femme adultère. Dans la version ivoirienne, le meurtrier involontaire est une jeune fille qui tue son amant par accident. Le cadavre est ensuite toujours trouvé par un homme. 
ne peuvent qu'être étoffés arbitrairement, de l'intérieur, par augmentation du nombre des protagonistes (quatre fils ou plus) ou une multiplication des actions accomplies par chacun d'eux. L'astuce pour faire durer le récit ne lui confère pas un sens, elle n'en change pas la temporalité. On peut d'ailleurs aisément constater, d'après les versions anciennes, que les conteurs ne se risquent pas à cet exercice, sauf à vouloir lasser leur auditoire en poussant la formule à l'extrême dans un « conte-attrape » où il ne se passe rien (par exemple la traversée d'un pont par un troupeau de moutons (T. 2080 AT, 2300 ATU).

Le rapport au temps de chacune des deux catégories de contes hâtivement répartis en « longs » (contes merveilleux et contes nouvelles) et «brefs », ou « courts » (contes d'animaux, du Diable dupé ou facétieux) correspond à deux projets différents et complémentaires: construction individuelle et sociale d'une part, divertissement de l'autre. L'un montre la nécessité et la possibilité de dépassement des oppositions, l'autre en fait son objet spécifique, il délivre sa leçon par l'exposé de l'échec. En schématisant encore, on avancera que le conte merveilleux dit ce qui doit être alors que le conte facétieux dit ce qui est et dont on s'accommode, faute de mieux. Il est le récit du dysfonctionnement, des failles de l'échange, des conflits à l'infini, des entorses (volontaires ou non, naïves ou malhonnêtes) aux lois du groupe. Il explore les arrangements provisoires avec des êtres incapables de prendre en compte l'altérité, construits « en marge du symbolique » (Chardenet 2OII) et dont les pulsions et désirs se réalisent en dépit de la loi et de la coutume. Toutes choses difficilement vécues dans le réel et difficiles à dire (les sous-entendus sont meurtriers) mais dont le récit partagé permettra l'acceptation au point d'en rire ensemble pourvu qu'on y mette les formes: qu'on en fasse un conte c'est-à-dire une œuvre, dans la proximité chaleureuse de l'oralité directe et susceptible de s'inscrire dans le temps long de la tradition. Parangon du récit bref, théoriquement opposée au genre conte par son autonomie, l'anecdote est le parfait exemple de l'art de dire vite et bien mais aussi de dire ensemble puisque sa construction « en pointe » délègue à l'auditeur la tâche essentielle et temporellement infime d'établir ce « court circuit de la pensée » (Bastide I970) qui fait jaillir le rire collectif suivi de la satisfaction procurée par cette connivence. ${ }^{16}$

Comme le souligne Louis Marin dans l'introduction d'un ouvrage posthume sur la transmission des contes en Lorraine (I964: I7-24), les folkloristes ne nous ont pas laissé d'informations suffisantes pour qu'il soit possible de poser fermement cette autre analogie qui relie le temps (bref) du conte facétieux et le moment où il est donné. Mais certains témoignages corroborent l'expérience que nous avons des récits plaisants (blagues ou autres narrations brèves à visée comique ou satirique). Ils nous montrent que les histoires brèves se glissent aisément ou font irruption dans les instants de rupture de rythme, les pauses durant le travail, les moments où l'on attend, la fin de quelque chose comme par exemple celle des

I6. Le rire est amené différemment par le conteur et par l'humoriste qui doit le provoquer à intervalles réguliers très rapprochés. Lors du Festival de contes de Fumel, en Lot-et-Garonne, en 2006, un conteur professionnel qui avait participé à un Festival du rire ou de l'humour, soulignait l'incompatibilité de cette règle avec le travail du conteur. Par ailleurs les Catch Tales, ou Contes-attrape permettent au narrateur fatigué de mettre fin à sa prestation. 
repas festifs... Dans les temps consacrés à conter, comme les veillées d'autrefois ou les spectacles contemporains, le conteur les introduit pour faire diversion, soit comme parenthèses soit comme épisodes visant à alléger l'atmosphère grave des récits plus profonds, soit pour « souffler » lui-même un instant. ${ }^{17} \mathrm{Il}$ module la qualité du temps passé ensemble et décide de sa quantité.

C'est donc bien le conteur, et non le récit lui même, qui façonne le temps consacré au récit oral et à l'écoute. Il est le maître du temps collectif engendré par la parole vivante. Entre les contes merveilleux dont les éléments sont organiquement ${ }^{18}$ assemblés et les formes narratives composées à partir d'unités brèves, il semble que quelque chose d'essentiel se soit déplacé. Ce n'est plus le héros marchant vers son destin qui crée et rythme le temps du récit, ni les formulettes qui le scandent, c'est le narrateur lui-même qui prend en charge la construction de la temporalité: non pas le banal assemblage des chaînes, mais plus largement celui d'un ensemble formé par toutes les histoires racontées à un même auditoire en un moment unique et non réitérable. Jouant avec les outils de l'art narratif traditionnel, avec son intuition, sa sensibilité et ses techniques personnelles, le conteur ou la conteuse alterne le grave et le léger, crée l'attente, l'enthousiasme, ou la crainte qu'il libère parfois en déclenchant le rire. Coupant le souffle de son public en suspendant sa parole, la reprenant à son gré sur un autre ton, il montre que le conte est bien un acte oral, produit de l'activité d'un conteur (Tenèze I975; Bru 20I2). Ne faut-il pas l'envisager dans le cadre plus large de la « contée » selon l'expression très juste des conteurs contemporains.

I7. André Lagarde à propos des nombreux contes merveilleux dits à ses enfants par son père à la veillée familiale dans les Pyrénées ariégeoises dans les années I930, écrit « Mas Joan de l'ors èra lo que nosautres aumàvem mai. Benlèu perque lo rire i costejava la paur e la realitat lo sòmi, que lo sublim s'i mesclava al grotèsc, lo lirisem a l'épopèia... »: (Mais Jean de l'ours était celui que nous aimions le plus. Peut-être parce que le rire y côtoyait la peur et la réalité le rêve, que le sublime s'y mêlait au grotesque, le lyrisme à l'épopée...) (Lagarde 2005: 39I). I8. Paul Delarue avait avancé la notion d'organisme pour évoquer le mode de composition des contes merveilleux et l'interdépendance de ses éléments. 


\section{Références bibliographiques}

AARne, Antti (I9IO): Verzeichnis der Märchentypen. Folklore Fellows' Communications 3. Helsinki : Suomalainen Tiedeakatemia.

AArne, Antti ; Stith Thompson (I96I): The Types of the Folktale. A Classification and Bibliography. Folklore Fellows' Communications I84. Helsinki : Suomalainen Tiedeakatemia.

ATU: voir Uther 2004.

BASTIDE, Roger (I970): « Le rire et les courts-circuits de la pensée ». Dans Mélanges offerts à Claude Lévi-Strauss. The Hague-Paris: Mouton, p. 953-963.

BELMONT, Nicole (I985): «Orphée dans le miroir du conte merveilleux ». L'Homme num. 93 (I985): 59-82. [Repris dans Belmont 2010: I83-205].

- (I993) : «Conte merveilleux et mythe latent ». Ethnologie française I (I993): 748I. [Repris dans Belmont 20IO: 206-2I8].

- (2004) : «La Fille aux trois galants. Nouvelle littéraire et composition en miroir ». Cahiers de littérature orale num. 56 (2004) : 67-8I. [Repris dans Belmont 2OIO: 278-290].

- (20I0): Mythe, Conte, Enfance. Les écritures d'Orphée et de Cendrillon. Anthropologie du monde occidental. Paris: L'Harmattan.

BRU, Josiane (2005) : « Du T I200 au I999, 'l'Autre' grande section de l'AarneThompson ». Cahiers de littérature orale num. 57-58 (2005): 43-76.

— (2007-2008) : « Des récits sans mémoire ou comment Jean resta garçon ». Estudos de literatura oral num. I3-I4 (2007-2008): 57-7I.

— (20I2) : «Qu'est-ce qu'un conte populaire? Un renversement complet de la perspective: Le Catalogue du conte populaire français à l'épreuve du terrain». Rabaska. Revue d'ethnologie de l'Amérique française num.Io (2012): 97-I23.

— (à paraître): «La légende comme récit bref et les limites du conte ». Dans «Entre conto e lenda ». Estudos de literatura oral. Actes des 5e rencontres du GRENO (20IO) à Torre des Vargens.

ChARDENET, Virginie (20Io): Destins de garçons en marge du symbolique. Les avatars de Jean le Sot. Paris: José Corti.

LAGARDe, André (2005) : Contes occitans. Tolosa : Edicions de l'Escòla Occitana [Nouvelle édition sous le titre Les secrets de las bèstias. Tolosa : Letras d'Oc, 2OI4].

LAJARTE, Philippe de (I974) : «Du conte facétieux considéré comme genre : esquisse d'une analyse structurale ». Ethnologie française num. IV, 4 (I974) : 3I9332.

MARIN, Louis (I964): Les contes traditionnels en Lorraine. Institutions de transfert des valeurs morales et spirituelles. Paris: Imprimerie Jouve.

Morin, Violette (I966): «L'histoire drôle». Communications num. 8 (I966): Io2II9. (Recherches sémiologiques. L'analyse structurale du récit).

Moser-RATH, Elfriede (I977) : «Anekdote ». Dans Enzyklopädie des Märchens I, coll. 528-54I.

Pelen, Jean-Noël (I994): Le conte populaire en Cévennes. GBP. Paris: Payot. 
Josiane Bru

RÖHRICH, Lutz (I990) : «La morale de l'immoralité. A mi-chemin entre la farce et l'histoire moralisante ». Dans Véronika GöröG-KARADY (éd.): D'un conte à l'autre. La variabilité dans la littérature orale. Paris: CNRS, p. 3I5-325.

SÉBILlot, Paul (I9I3): «Littérature orale anecdotique ». Revue des Traditions populaires num. 28-I (I9I3): 289-29I.

SIMONSEN, Michèle (2003-2004) : «Un parent pauvre de la littérature orale. Réflexion sur le conte facétieux ». Estudos de literatura oral num. 9-Io (20032004): 253-266.

TAYLOR, Archer (I970): "The Anecdote: A Neglected Genre ». Dans Jerome MANDel; Brun A. Rosenberg (éds.) : Medieval Literature and Folklore Studies. Essays in Honor of Francis Lee Utley. New Brunswick: University Press, p. 223-228.

TENÈze, Marie-Louise (I975) : «Littérature orale narrative ». Dans L'Aubrac, Etude ethnologique, linguistique, agronomique et économique d'un établissement humain, tome V, Ethnologie contemporaine III. Paris: CNRS, p. 32-I64.

— (I976) : Le conte populaire français, Tome troisième [Contes d'animaux]. Paris: G. -P. Maisonneuve et Larose.

— (2000): Le conte populaire français. Contes-nouvelles. Références de l'ethnologie. Paris: Éditions du CTHS.

- (2004): Les contes merveilleux français. Recherche de leurs organisations narratives. Avec la collaboratin de Josiane Bru. Paris: Maisonneuve et Larose.

Tenèze, Marie-Louise ; Georges Delarue (éds.) (200o) : Nannette Lévesque, conteuse et chanteuse du pays des sources de la Loire. Le langage des contes. Paris : Gallimard.

UTHER, Hans-Jörg (2004): The Types of International Folktales. A Classification and Bibliography. Folklore Fellows' Communications 284-285-286. Helsinki : Suomalainen Tiedeakatemia. 